\title{
Downregulation of long non-coding RNA SNHG7 protects against inflammation and apoptosis in Parkinson's disease model by targeting miR-425- 5p/TRAF5/NF-kB axis
}

\section{Shan Yu}

China-Japan Union Hospital of Jilin University

Xueshibojie Liu

Second Hospital of Jilin University

Duo Yu

Second Hospital of Jilin University

Changyong $E$

China-Japan Union Hospital of Jilin University

Jinghui Yang ( $\nabla_{\text {jinghui2393@163.com ) }}$

China-Japan Union Hospital of Jilin University

\section{Research}

Keywords: Parkinson's disease, SNHG7, miR-425-5p, inflammation, TRAF5

Posted Date: May 20th, 2020

DOI: https://doi.org/10.21203/rs.3.rs-29077/v1

License: (c) (i) This work is licensed under a Creative Commons Attribution 4.0 International License.

Read Full License 


\section{Abstract \\ Background}

Accumulated evidence has established that long non-coding RNA (IncRNA) is involved in the progress of Parkinson's disease (PD). SNHG7, a novel IncRNA, has been found to play a key role in tumorigenesis. However, the SNHG7 expression and its functional effects on PD remain uncharted.

\section{Methods}

RT-PCR was used to detect the expression of SNHG7, miR-425-5p and inflammatory cytokines in the plasma of PD patients and the healthy controls. Rotenone (Rot) was adopted to construct PD models in SD rats and SH-SY5Y cells, respectively. Gain- and loss- of functions of SNHG7 or miR-425-5p were conducted. The expression levels of Caspase3, tyrosine hydroxylase (TH), Iba1 in SD rat striatum was measured via immunohistochemistry and Western blot. Additionally, the expressions of inflammatory cytokines (IL-1 $\beta$, IL-6, TNF-a) and oxidative stress factors (MDA, SOD, GSH-PX) in the brain tissues were examined using RT-PCR and ELISA. Moreover, the protein levels of TRAF5, l-kB, NF-KB, HO-1, Nrf2 were detected via Western blot. Bioinformatics was applied to predict the targeting relationship between SNHG7, miR-425-5p and TRAF5. Dual luciferase activity assay and RNA immunoprecipitation (RIP) assays were carried out to verify their interactions.

\section{Results}

SNHG7 was found up-regulated in PD patients while miR-425-5p expression was down-regulated (compared to healthy donors). Meanwhile, SNHG7 level was positively correlated with the level of inflammatory cytokines in PD patients. Functional experiments confirmed that SNHG7 downregulation or miR-425-5p overexpression attenuated neuronal apoptosis in the Rot-mediated PD model, TH-positive cell loss and microglia activation by mitigating inflammation and oxidative stress. Mechanistically, SNHG7 served as a competitive endogenous RNA (ceRNA) by sponging miR-425-5p and promoted TRAF5 mediated inflammation and oxidative stress.

\section{Conclusion}

Inhibition of SNHG7 ameliorated neuronal apoptosis in PD through relieving miR-425-5p/TRAF5/NF-KB signaling pathway modulated inflammation and oxidative stress.

\section{Introduction}

As a typical neurodegenerative disorder disease, Parkinson's disease (PD) inflicts serious damage to the patients' quality of life. Clinically, PD presents symptoms like resting tremors and muscle rigidity. 
Pathologically, the disease is characterized by the degeneration or progressive loss of dopaminergic neurons in the substantia nigra pars compacta and striatum of mid-brain, together with the presence of Lewy bodies (mainly composed of a-synuclein) [1]. A series of studies have confirmed that genetic susceptibility, oxidative stress, drug toxicity, and environmental triggers are strongly implicated in PD's pathogenesis [2-3]. In recent years, as increasing research has focused on the pathogenic mechanism underlying PD, the causal genes of PD and non-coding RNAs (such as IncRNA and miRNA) that regulate these genes have attracted wider attention [4, 5]. Thus, an improved understanding of IncRNA in the PD pathogenesis/ carries great significance, promising to provide new insights for early diagnostic indicators and effective therapeutic targets of PD.

According to recent studies, IncRNA regulates a variety of inflammations by targeting miRNA [6, 7]. For example, SNHG16 regulates CCL5 expression by competitively binding to miR-146a-5p; SNHG16 overexpression aggravates the apoptosis, as well as inflammation of LPS-induced acute pneumonia in WI-38 cells [8]. Moreover, FGD5-AS1 affects the occurrence and development of periodontitis by regulating the miR142-3p/SOCS6/NF-KB pathways [9]. In PD, similar mechanisms for IncRNA regulation are also available. For example, IncRNA-p21 is up-regulated in a PD model of MPP-induced neuronal damage in SH-SY5Y cells. Besides, IncRNA-p21 upregulates TRPM2 expression by targeting miR-625 [10]. As a group of IncRNAs, the SNHG family is considered as key regulators in PD. For instance, SNHG1 is upregulated in PD and over-expressed SNHG1 promotes MPP-induced SH-SY5Y cell apoptosis [11]. In another sduty, SNHG1 regulates the p27/mTOR pathway by competitively binding to miR-221/222. SNHG1 knockdown mitigates MPP-induced PD cytotoxicity [12].

SNHG7 also belongs to IncRNAs, which is located on chromosome 9q34.3. Besides, SNHG7 has been found to exert oncogenic effects on multiple cancers, including colorectal cancer [13], pancreatic cancer [14], and breast cancer [15] mainly by modulating the proliferation, migration, invasion and apoptosis of tumor cells. Additionally, SNHG7 could inhibit high glucose-induced cell proliferation, migration and angiogenesis [16], and also alleviate osteoarthritis through modulating the proliferation, apoptosis and autophagy of chondrocytes [17]. However, the role of SNHG7 in PD progression remains to be further verified.

TRAF5 is a member of the tumor necrosis factor receptor-associated factor (TRAF) family, which has been discovered recently. Like other TRAF family members, TRAF5 also has a ring finger domain, so it displays E3 ubiquitin Ligase viability. Existing studies have validated that TRAF5, as a cytoplasmic adapter, can activate the NF-KB signaling pathway through its receptors, and then work in nervous system inflammation, neuronal apoptosis, glial cell activation and other processes [18, 19]. At present, accumulating studies have highlighted that TRAF5, as a targeting molecule for various miRNAs, is directly involved in the regulation of related diseases. For example, in gastric cancer cells, miR-135a regulates the NF-KB pathway by targeting TRAF5. MiR-135a overexpression suppresses the NF-KB pathway, thereby inhibiting gastric cancer cell migration [20]. 
In the present study, we found that SNHG7 was significantly overexpressed in the serum of PD patients and also positively correlated with the levels of proinflammatory cytokines (IL-1 $\beta$, IL-6, TNF-a). Additionally, we conducted bioinformatics analysis through Starbase (http: //starbase.sysu.edu.cn) and found that miR-424-5p shared the binding sites with SNHG7 and TRAF5. Interestingly, miR-424-5p was markedly downregulated in the serum of PD patients and had negative correlations with IL-1 13 , IL- 6 , and TNF-a. Therefore, we supposed there is a regulatory network of SNHG7-miR-424-5p-TRAF5 in PD development.

\section{Materials}

\subsection{Clinical samples}

A total of 56 participants were recruited from China-Japan Union Hospital of Jilin University: 36 surgically treated PD patients and 20 healthy donors who have undergone related examinations as required. All the recruiters provided written informed consent. Moreover, this research has garnered approval from the Ethics Committee of China-Japan Union Hospital of Jilin University Hospital. All the plasma samples (fasting elbow vein blood, $10 \mathrm{~mL}$ ) were obtained from the recruiters in the morning. After that, the samples were subjected to experimental examination after centrifugal separation.

\subsection{Animals and treatment}

A total of 45 Sprague-Dawley (SD) rats (male, $240 \sim 260 \mathrm{~g}$, clean grade) were obtained from the Experimental animal center, Bethune Medical Department, Jilin University. All of the rats were kept under standard laboratory conditions. All animal experiments had been given approval from the Animal Experiment Ethics Review Committee of China-Japan Union Hospital of Jilin University and conducted following the guidelines for laboratory animal care and use of the National Institutes of Health $\mathrm{NIH}$ publication number 8023, 1978 revision). A total of 45 SD rats were randomly divided into 3 groups, namely the Sham group, the Rot + LV-si-NC group, and the Rot + LV-si-SNHG7 group. For the Sham group: sunflower oil was subcutaneously injected into the back of SD rats for 42 days $(1 \mathrm{ml} / \mathrm{kg} /$ day). For the Rot + LV-si-NC group and Rot + LV-si-SNHG7 group: rotenone (MedChemExpress, HY-B1756) was subcutaneously injected at the rats' back for 42 days consecutively $(1.5 \mathrm{mg} / \mathrm{kg} / \mathrm{day})$. The small inference RNA (siRNA) lentivirus vector targeting SNHG7 (LV-si-SNHG7) or LV-si-NC (GeneChem Co. Ltd, Shanghai, China) were inserted into the linearized vector GV115 to construct the siRNA recombinant lentiviral vector. Two days before Rot treatment, the rats were deeply anesthetized via isoflurane in oxygen and nitrous oxide, and then positioned on a stereotactic frame (Stoelting, Wood Dale, IL). The skull surface was exposed with a hole drilled to locate the needle. Hamilton syringes ( $5 \mu \mathrm{L}, 33$-gauge needle) were applied here to inject the rats with $1 \mu \mathrm{L} /$ side of recombinant LV-si-SNHG7 or LV-si-NC into the dorsal hippocampus (anterior - 5.5; lateral + 1.6; dorsoventral - 7.5 from bregma) $(0.2 \mu \mathrm{L} / \mathrm{min}, 10 \mathrm{~min})$. The behavioral evaluation was carried out on day 0,14 , and 42 . Rats were sacrificed by decapitation following the behavioral evaluation on the 42th day. After anesthetization and perfusion, the rat brains were collected, washed and frozen in liquid nitrogen. They were stored at $-80^{\circ} \mathrm{C}$ for further use. 


\subsection{Behavioral assessment}

Using computer-aided infrared motion detection system (Institute of Materia Medica, Chinese Academy of Medical Sciences, China), spontaneous sports activities were measured. The rats were placed in transparent plexiglass bottles ( $40 \mathrm{~cm}$ across, $13 \mathrm{~cm}$ high), making them get used to the environment 5 minutes prior to the experiment. Then the number of horizontal and vertical movements was recorded in 10 minutes.

On the carousel experiment: First, the rats were placed on a fixed rod ( $3 \mathrm{~cm}$ across) for 30 seconds, during which any fallen animals were placed back on the rod. Next, the conditioned response of the rats was made to formulate in the following 120 seconds at a constant speed of $15 \mathrm{RP} / \mathrm{m}$. For those failed to formulate the response, two additional chances were granted. During the fatigue time after the last training, the rats were placed on the rod at a constant speed of $15 \mathrm{RP} / \mathrm{m}$ ( 120 seconds per time) to evaluate their motor skills. Measurements were recorded three times at 30-minute intervals per round.

On the bevel experiment: To begin with, the rats were placed on the bevel rough surface at an angle of $60^{\circ}$. Then, the duration during which the rats of each group stay on the bevel was recorded respectively. When the rat stayed on the slope for over 3 minutes, it was recorded as 180 seconds. Three experiments were performed on each animal.

\subsection{Immunohistochemical staining}

The brain slices were put in a methanol solution $\left(0.3 \% \mathrm{H}_{2} \mathrm{O}_{2}\right)$ and got incubated $\left(37^{\circ} \mathrm{C}, 15 \mathrm{~min}\right)$ to inactivate the endogenous peroxidase. Next, the sections were rinsed with PBS $(0.01 \mathrm{~mol} / \mathrm{L}, \mathrm{pH} 7.4)$ for $5 \mathrm{~min}(\times 3)$, and incubated with $10 \%$ normal goat serum $\left(37^{\circ} \mathrm{C}, 15 \mathrm{~min}\right)$. When the goat serum was removed, the slices were added with rabbit antibodies including anti-TH (1: 200; Abcam, ab75875, MA, USA), anti-Caspase3 (1:200, Abcam, ab13847, MA, USA) and kept in the wet box $\left(4{ }^{\circ} \mathrm{C}\right)$ overnight. Then, the samples were rinsed by PBS for $5 \mathrm{~min}(\times 3)$, added with biotin-labeled goat anti-mouse IgG (1: 200, Beijing Zhongshan Biotechnology Co., Ltd.), and incubated $\left(37^{\circ} \mathrm{C}, 30 \mathrm{~min}\right)$. Afterwards, we washed them with PBS for $5 \mathrm{~min}(\times 3)$, added ABC solution (Beijing Zhongshan Biotechnology) Co., Ltd.), and incubated them at $37^{\circ} \mathrm{C}$ for $30 \mathrm{~min}$. Further, the sections were rinsed with PBS for $5 \mathrm{~min}(\times 3)$ and kept for DAB color development for $10 \sim 15 \mathrm{~min}$. Lastly, the sections were dehydrated with gradient alcohol, made transparent via xylene, sealed with neutral gum, and ultimately observed under an optical microscope.

\subsection{Immunofluorescence}

The frozen sections were taken, hydrated with $0.01 \mathrm{~mol} / \mathrm{L}$ phosphate buffer for $20 \mathrm{~min}$ and repaired with citric acid in the microwave for about 5 min. The membranes were ruptured with $0.3 \%$ Triton $\mathrm{X}-100$ for $30 \mathrm{~min}$, washed with $0.01 \mathrm{~mol} / \mathrm{L}$ phosphate buffer for $5 \mathrm{~min}(\times 3)$, blocked with antigen blocking solution (1 h). Next, the rabbit anti-Iba1 antibody (1: 200; Abcam, ab178846, MA, USA) was added dropwise for incubation overnight $\left(4^{\circ} \mathrm{C}\right)$. Then, the specimens were subjected to $0.01 \mathrm{~mol} / \mathrm{L}$ phosphate buffer washing for $5 \mathrm{~min}(\times 3)$, added with goat anti-rabbit fluorescent IgGII antibody labeled with AlexaFluor 555, and incubated $\left(37^{\circ} \mathrm{C}, 1 \mathrm{~h}\right)$ in the dark room. We washed the sections with $0.01 \mathrm{~mol} / \mathrm{L}$ phosphate buffer for 
5 min ( $\times 4$ ), mounted them via the medium with 4'6-diamino-2-phenylindole (DAPI) and ultimately put them under fluorescent microscope observation. Three slices of brain tissue at approximately the same level per rat were taken to observe Iba 1 positive microglia around the substantia nigra at five randomly selected fields $(\times 200)$ and calculated for the number of positive cells.

\subsection{Cell culture and transfection}

Human dopaminergic neuroblastoma SH-SY5Y cells and microglia BV2 cells were obtained from the Chinese Academy of Sciences (Shanghai, China) and cultured in Dulbecco's modified Eagle's mediuHam's Nutrient Mixture F-12 (DMEM/F12; Invitrogen, Carlsbad, CA) supplemented with $10 \%$ fetal bovine serum (FBS), penicillin and streptomycin ( $1 \%$ in humidified air $\left(37^{\circ} \mathrm{C}, 5 \% \mathrm{CO}_{2}\right)$. Then, the SH-SY $5 \mathrm{Y}$ cells were further cultured in neuron medium (ScienCell Research Laboratories, Carlsbad, $\mathrm{CA})\left(37^{\circ} \mathrm{C}, 5 \% \mathrm{CO}_{2}\right)$, added with $1 \%$ neuron growth agent and $1 \%$ penicillin and streptomycin.

Rotenone was dissolved in dimethyl sulfoxide (DMSO). The final DMSO solution concentration was $0.01 \%$. SH-SY $5 Y$ cell damage was induced using rotenone $(500 \mathrm{nM})$ for $24 \mathrm{~h}$ and BV2 cell activation was induced by lipopolysaccharide (Sigma, $10 \mu \mathrm{g} / \mathrm{mL}$ ). Twenty-four hours before transfection, cells (logarithmic growth phase) were seeded into a 96-well microplate to achieve a $60-80 \%$ fusion rate. Then the expressing vectors of SNHG7 overexpression plasmids, si-SNHG7, miR-425-5p mimics and their negative controls (GenePharma, Shanghai, China) were transferred into SH-SY5Y and BV2 cells using Lipofectamine 2000 Reagent (ThermoFisher Scientific, CA, USA) in line with the manufacturer's recommendations. The cells were harvested for further experiments after 48 hours' transfection.

\section{7 quantitative real-time PCR (qRT-PCR)}

First, total RNA was extracted from human plasma, rat tissues and SH-SY5Y cells using TRIzol reagent (Invitrogen, Carlsbad, CA, USA). After being checked for purity, it was then reverse transcribed into cDNA via RevertAid First Strand cDNA Synthesis Kit (Thermo Fisher Scientific, Waltham, MA, USA).

Subsequently, we used SYBR ${ }^{\circledR}$ Premix-Ex-Taq ${ }^{\text {TM }}$ (Takara, TX, USA) and ABI7300 system for quantitative reverse transcription-polymerase chain reaction (qRT-PCR). The total volume of the PCR system was $30 \mu \mathrm{L}$ with each sample containing $300 \mathrm{ng} \mathrm{cDNA}$. Under the amplification procedure, the first denaturation lasted 10 minutes $\left(95^{\circ} \mathrm{C}\right)$, and the following 45 cycles were: $95^{\circ} \mathrm{C}$ ( 10 seconds), $60{ }^{\circ} \mathrm{C}(30$ seconds), and $85^{\circ} \mathrm{C}$ (20 seconds). All fluorescence data were converted into relative quantification, seeing $\beta$-actin as the endogenous control for SNHG7, and U6 for miR-425-5p. Additionally, the $2^{-\Delta \Delta C t}$ method was used for statistics with all experiments repeated in triplicate. The RNA primer sequences were as follows: SNHG7 Forward: 5'-aaatgtcagcagtgccagtg-3', Reverse, 5'-gatggggtttcaggcagttg-3'; miR425-5p, Forward, 5'-TGCGGAATGACACGATCACTCCCG-3', Reverse, 5'-CCAGTGCAGGGTCCGAGGT-3'; U6 Forward, 5'-TGCGGGTGCTCGCTTCGGCAGC-3', Reverse, 5'-CCAGTGCAGGGTCCGAGGT-3', $\beta$-actin Forward, 5'-CAGAGCCTCGCCTTTGCC-3', Reverse®5'-GTCGCCCACATAGGAATC-3'.

\subsection{Western blot}


When rat brain tissues or cells were ready, we removed the medium and added protein lysate (Roche) to separate the total protein. Next, $50 \mathrm{~g}$ total protein was added to $12 \%$ polyacrylamide gel for electrophoresis (100V, $2 \mathrm{~h}$ ). Then, the protein samples were electroblotted onto polyvinylidene fluoride (PVDF) membranes. After blocking with $5 \%$ skimmed milk powder ( $1 \mathrm{~h}$, room temperature), the membranes were washed 3 times with TBST for 10 min each time. Afterwards, the membranes were incubated $\left(4^{\circ} \mathrm{C}\right)$ overnight, followed by an incubation with antibodies as follows: anti-tyrosine hydroxylase (Th) (1: 1000, Abacam, ab75875, MA, USA), anti-TRAF5 (1: 1000, Abacam, ab137763, MA, USA), anti-I-kB (1: 1000, Abacam, ab32518, MA, USA), p-NF-kB (phospho S536) (Abcam, ab86299, 1: 1000), anti-NF-kB (1: 1000, Abacam, ab32536, MA, USA), anti-Nrf2 (1: 1000, Abacam, ab31163, MA, USA), anti-HO-1 (1: 1000, Abacam, ab13248, MA, USA) ), anti-Bax (1: 1000, Abacam, ab32503, MA, USA), antiBcl2 (1: 1000, Abacam, ab182858, MA, USA), and anti-Caspase3 (1: 1000, Abacam, ab13847, MA, USA), anti-Iba1 (1: 200; Abcam, ab178846, MA, USA). Washed with TBST, the membranes were incubated with horseradish peroxidase (HRP) labeled anti-rabbit or anti-mouse secondary antibody (concentration 1: 3000 ) for $1 \mathrm{~h}$. Then, we washed the membrane 3 times with TBST (10 min each). Finally, Western blot reagent (Invitrogen) was applied for color imaging, using Image $\mathrm{J}$ for analyzing the gray value of each protein.

\subsection{MTT Assay}

$100 \mu \mathrm{L} \mathrm{SH-SY5Y} \mathrm{cells} \mathrm{(logarithmic} \mathrm{growth} \mathrm{phase)} \mathrm{were} \mathrm{seeded} \mathrm{into} \mathrm{a} \mathrm{96-well} \mathrm{plate} \mathrm{(about} 4 \times 10^{3}$ /well) and incubated at $37^{\circ} \mathrm{C}$, with $5 \% \mathrm{CO}_{2}$ and $100 \%$ humidity for 24 hours. Afterward, we added an equal volume of PBS to the control group (5 repetitive wells each group) and continued to add $50 \mu \mathrm{L}$ MTT $(5 \mathrm{~g} / \mathrm{L})$ after 24 hours of incubation. 4 hours later, the supernatant was discarded and $150 \mu \mathrm{L}$ DMSO was added into each well. Then the plate was placed in a platform rocker for shaking for 1 hour. When the crystals were dissolved, a microplate reader was utilized to detect the OD value in each well $(570 \mathrm{~nm}$ wavelength).

\subsection{Measurement of malondialdehyde (MDA), superoxide dismutase (SOD) and glutathione peroxidase (GSH-PX)}

After the brain tissues were weighed, 9 times volumes of cold saline (containing protease inhibitors) were added for homogenization Then, the samples subjected to a centrifugation at $3000 \mathrm{xg}$ for 20 minutes $\left(4^{\circ} \mathrm{C}\right)$. After that, the supernatant was collected. Regarding Rot-treated SNHG7 cells, the cell supernatant was collected according to the group, and centrifuged at $1000 \times \mathrm{g}\left(10 \mathrm{~min}, 4^{\circ} \mathrm{C}\right)$. Next, the supernatant was collected. SOD and GSH content in brain tissues or cells were determined respectively with the help of Superoxide dismutase (SOD), glutathione peroxidase (Glutathione peroxidase (GSH-PX) and malondialdehyde (MDA), a procedure carried out under the requirements of the kit. The above oxidative stress factor detection kits were purchased from Nanjing Jiancheng Bioengineering Institute (Nanjing Jiancheng Bioengineering Institute, China).

\subsection{Enzyme linked immunosorbent assay (ELISA)}


To begin with, the test tube was filled with $10 \mathrm{~mL}$ venous blood. Standing for 1 hour at room temperature, the sample was subject to a centrifugation (3000 r/min, 5-10 minutes). Next, the upper serum was collected and relocated to the refrigerator $\left(-20^{\circ} \mathrm{C}\right)$ for overall detection. Following that, the SD rat brain tissues were weighed, shredded, and added with 12 volumes of normal saline. The brain tissues in normal saline were then transported to a homogenizer for thorough mixing and crushing to deliver a brain tissue homogenate. Furthermore, the brain tissue homogenate was centrifuged $(6000 \mathrm{r} / \mathrm{min}, 15 \mathrm{~min}$, $4{ }^{\circ} \mathrm{C}$ ), and then the supernatant was collected for detection. Then, the supernatant of Rot-treated SNHG7 cells was collected based on the group. After a 10 min centrifugation $\left(1000 \times \mathrm{g}, 4^{\circ} \mathrm{C}\right)$, the supernatant was collected as required.

All experimental steps conformed to the instructions provided by the ELISA kit in determining the content of IL-1 $\beta$, IL-6, TNF- $a$ and lactate dehydrogenase (LDH) release. The test kits were obtained from Nanjing Jiancheng Bioengineering Institute (NanJing JianCheng Bioengineering Institute, China).

\subsection{Luciferase reporter assay}

The DNA sequences amplified were cloned into the pmirGLO dual luciferase vector (Promega, Madison, WI, USA) to generate wild-type (WT) SNHG7 3-UTR, mutant (MUT) SNHG7 3-UTR, wild-type (WT) TRAF5 3UTR, mutant (MUT) TRAF5 3-UTR reporter vectors. The cells were seeded into a 24-well plate overnight. Then Lipofectamine 2000 was used to transfect SNHG7-WT, SNHG7-MUT, TRAF5-WT, TRAF5-MUT reporter vectors and miR-425-5p mimics (Invitrogen, Carlsbad, CA, USA) into SH-SY5Y cells. Through the dual-luciferase reporter system (Promega, Madison, WI, USA), luciferase activity was determined after $48 \mathrm{~h}$ of transfection.

\subsection{RNA immunoprecipitation (RIP) assay}

To determine the relations between SNHG7 and miR-425-5p, miR-425-5p and TRAF5, Magna-RNA combined with the binding protein immunoprecipitation kit (millipore, Bedford, Ma, USA) was used to conduct the RIP test. The SH-SY5Y cells (transfected with miR-424-5p or miR-NC) at $80 \%$ confluence were collected and lysed in a complete RIP lysate buffer. Then the cell lines were co-immunized with RIP buffer containing magnetic beads with anti-argonaute 2 (Ago2) antibody (Millipore) or negative control healthy rat IgG (Millipore). The immunoprecipitated RNA was isolated and the relative enrichment of SNHG7 and TRAF5 in the lysates was examined by qRT-PCR.

\subsection{Statistical analysis}

The data analysis was carried out via SPSS software (version 20.0, Chicago, IL, USA). The data were displayed as mean \pm standard deviation (SD). Student' $t$-test, along with $\chi 2$ were employed to access the statistical differences between the two groups. The correlation relationship was determined by Pearson correlation test. $P<0.05$ was regarded as statistically significant.

\section{Results}




\subsection{SNHG7 and miR-425-5p expression characteristics in PD patients}

Aiming at determining the SNHG7 and miR-425-5p expressions in PD patients, qRT-PCR was employed to detect the expressions in 36 PD patients and 20 healthy donors. The results indicated that compared with the healthy donors, SNHG7 was up-regulated in PD patients plasma, while miR-425-5p was downregulated (Fig. 1A, B). Pearson correlation analysis exhibited that SNHG7 in PD patient plasma was negatively correlated with miR-425-5p expression (Fig. 1C). Furthermore, the IL-1 $\beta$, IL-6, and TNF-a relative expressions in the plasma were detected via ELISA. The results indicated that the levels of IL-1 $\beta$, IL- 6 , and TNF-a in PD patient plasma were obviously increased (Fig. 1D). By conducting Pearson correlation analysis, we found that SNHG7 was positively correlated with IL-1 $\beta$, IL-6, TNF-a in PD patient plasma, while miR-425-5p revealed a negative correlation with IL-1 $\beta, I L-6$, TNF-a (Fig. 1E, F). Therefore, the results suggested that both SNHG7 and miR-425-5p might play a role in PD.

\subsection{SNHG7 inhibition attenuated neuronal apoptosis in PD model in vivo and in vitro}

To better understand the biofunctions of SNHG7 on PD, we used Rot to construct a PD model in SD rats. Meanwhile, LV-si-SNHG7 was used to establish a rat model with downregulation of SNHG7. The spontaneous locomotor activity test, carousel experiment and bevel experiment were conducted to comprehensively assess the motor skills of rats. As Fig. 2A showed, the rats in the Rot + LV-si-NC group showed evident behavioral damage in comparison to the Sham group. However, the Rot + LV-si-SNHG7 group showed marked less behavioral damage than the Rot + LV-si-NC group (Fig. 2A). The figure of tyrosine hydroxylase $(\mathrm{TH})$ positive cells in substantia nigra (SN) was detected by immunohistochemistry, and it was found that Rot-induced TH positive cell number in SD rats was prominently reduced, while knocking down SNHG7 reversed Rot's effect on TH positive cell number in SD rats (Fig. 2B). Additionally, Western blot was applied to detect $\mathrm{TH}$ relative expression in the $\mathrm{SN}$ area. Results indicated that $\mathrm{TH}$ relative expression enhanced in the Rot + LV-si-SNHG7 group compared to the Rot + LV-si-NC group (Fig. 2C). Moreover, Caspase3 staining was used to detect the number of apoptotic neurons. As a result, more Caspase 3 positive staining cells were found in the the Rot + LV-si-NC group (compared with Sham group), however, SNHG7 knockdown obviously inhibited Caspase3 labeled apoptotic cells (Fig. 2D). To further verify the effect of SNHG7 in modulated rotenone induced neuronal damage, si-SNHG7 was used to establish a SNHG7-downregulated cell model in SH-SY5Y cells (Fig. 2E), which were also treated with Rot. Next, the cell viability, release of LDH and apoptosis were determined. The results showed that compared with control group, Rotenone significantly inhibited cell viability (Fig. 2F), promoted LDH expression (Fig. 2G) and enhanced apoptosis (Fig. 2H). However, downregulating SNHG7 increased cell viability, reduced LDH expression and cell apoptosis (Fig. 2F-H). The above results illustrated that SNHG7 knockdown exerted neuro-protective effects against rotenone induced PD both in vivo and in vitro. 


\subsection{SNHG7 inhibition attenuated inflammation and oxidative stress in PD models}

In the PD rat model, we further validated whether SNHG7 regulated PD's inflammation and oxidative stress. Here, immunofluorescence was performed to test lba1-labeled microglial cells. The findings suggested that microglial cell activation in the PD model was remarkably stimulated, while SNHG7 knockdown notably reduced microglial activation (Fig. 3A and B). Moreover, the proinflammatory cytokines in brain tissues was measured by ELISA. The results showed that the levels of IL-1 $\beta$, IL-6, and TNF-a were all overexpressed in the Rot + LV-si-NC group (compared with the Sham group), while SNHG7 inhibition attenuated the inflammatory responses in the PD model (Fig. 4A). Next, the expression of oxidative stress factors including MDA, SOD, and GSH-PX in brain tissues were also determined. We discovered that knocking down SNHG7 attenuated MDA level but enhanced SOD and GSH-PX level in the brain tissues, suggesting that SNHG7 downregulation inhibited oxidative stress response (Fig. 4B). Then, we examined the relative expression of TRAF5, I-KB, p-NF-KB, Nrf2, HO-1 using Western blot. TRAF5 and pNF-KB expressions in the Rot + LV-si-SNHG7 group were markedly down-regulated comparing to that of the Rot + LV-si-NC group, while the expression of I-KB, Nrf2 and HO-1 were elevated conspicuously (Fig. 4C). Similarly, we investigated the regulatory role of SNHG7 on the inflammation and oxidative stress in BV2 cells induced by LPS. ELISA results showed that SNHG7 inhibition repressed Rot-induced upregulation of inflammatory cytokines (IL-1 $\beta$, IL-6, and TNF-a) (Fig. 4D). After detecting the oxidative stress factors' expression, we reported that in Rot-treated cells, the relative expression of MDA raised significantly while SOD and GSH-PX were decreased. After suppressing SNHG7, the relative expression of MDA was distantly down but SOD and GSH-PX expressions was enhanced (Fig. 4E). Additionally, the expressions of TRAF5 and p-NF-KB in the LPS + si-SNHG7 group were greatly down-regulated compared with the LPS + si-NC group, while the expressions of I-KB, Nrf2, and HO-1 were significantly increased (Fig. 4F). The above results indicated that SNHG7 inhibition could relieve microglia mediated inflammation and oxidative stress via regulating TRAF5/l-kB/NF-KB pathway.

\subsection{SNHG7 targeted miR-425-5p}

To explore the downstream molecular mechanism of SNHG7, we analyzed the SNHG7 candidate targets through the StarBase database (http://starbase.sysu.edu.cn). Interestingly, miR-425-5p had the binding sites with SNHG7 (Fig. 5A). The dual luciferase reporter assay and RIP assay were applied to determine the interactions between SNHG7 and miR-425-5p. As the results were shown, miR-425-5p mimics reduced the luciferase activity of BV2 cells transfected with SNHG7 (wild-type), but exerted minimal effect on the luciferase activity of mutant SNHG7. Moreover, the enrichment of SNHG7 in anti-Ago2 antibody precipitation complex was remarkably increased following miR-424-5p mimics transfection. What's more, the miR-425-5p relative expression the PD model (both in vivo and in vitro) were declined, while downregulation of SNHG7 promoted miR-425-5p expression (Fig. 5D and E). The above results showed that SNHG7 may function as a ceRNA by sponging miR-425-5p. 


\subsection{SNHG7 overexpression inhibited miR-425-5p mediated neuroprotective effects}

To further investigate the SNHG7/miR-425-5p axis in PD, SH-SY5Y cells were transfected with miR-425-5p mimics and/or SNHG7 overexpressing plasmids. The results showed overexpression of miR-425-5p inhibited SNHG7 level (Fig. 6A), at the same time, SNHG7 overexpression suppressed miR-425-5p level (Fig. 6B). Next, the cell viability of SH-SY5Y cells was tested by MTT assay, which showed that the cell viability in the Rot + miR-425-5p group was apparently enhanced (compared with Rot group), while supplementation of the SNHG7 overexpressing plasmids resulted in a remarkable decrease in cell viability (Fig. 6C). Additionally, ELISA assay was performed to ascertain the LDH level. We found that miR-425-5p mimics obviously reduced LDH expression, which was significantly blocked following SNHG7 overexpression (Fig. 6D). Besides, the expression of apoptosis-related proteins (Bax, Bcl2, and Caspase3) were examined via western blot in each group, and results stated that miR-425-5p decreased the Rotinduced $\mathrm{Bax}$ and Caspase 3 overexpression and $\mathrm{Bcl} 2$ downregulation, nevertheless, compensation of SNHG7 reversed those effects (Fig. 6E). Hence, SNHG7 promoted rotenone mediated neuronal damage via inhibiting miR-425-5p.

\subsection{SNHG7 promoted TRAF5/NF-KB activation by competitively binding to miR-425-5p}

To further verify the underlying mechanism of SNHG7/miR-425-5p axis in regulating microglia activation, the relative expression of inflammatory factors and oxidative stress factors were detected by ELISA. It turned out that miR-425-5p overexpression inhibited the inflammatory cytokines and oxidative stress induced by LPS in BV2 cells, which were inhibited by SNHG7 (Fig. 7A and B). Next, the result of western blot showed that the TRAF5 and p-NF-KB expressions in the LPS + miR-425-5p group declined, while the I$\mathrm{KB}, \mathrm{Nrf} 2$ and HO-1 expressions increased in comparison to LPS group. However, SNHG7 reversed the effects induced by miR-425-5p (Fig. 7C, D). The above statistics indicated that SNHG7 promoted the activation of TRAF5/NF-KB pathway in microglia through competitively binding with miR-425-5p.

\section{7 miR-425-5p targeted TRAF5}

In an effort to reveal the downstream mechanism of miR-425-5p, the bioinformatics database Starbase (http://starbase.sysu.edu.cn) was employed to find the miR-425-5p downstream genes. In this study, TRAF5 was identified as an important target molecule towards miR-425-5p (Fig. 8A). To clarify whether miR-425-5p targeted TRAF5, the dual luciferase activity assay and RIP assay were conducted, which showed that miR-425-5p dramatically inhibited the TRAF5 WT luciferase activity, but exerted minimal effect on TRAF5 MUT. Moreover, the Anti-Ago2 RIP assay demonstrated that miR-425-5p mimics led to markedly high level of TRAF5 in the anti-Ago2 group (Fig. 8B, C). These findings showed that miR-425-5p could target TRAF5. 


\section{Discussion}

In the present study, we explored a novel network of SNHG7-miR-425-5p-TRAF5 axis in PD progression. Our data showed that downregulation of SNHG7 markedly repressed the inflammation and oxidative stress through upregulating miR-425-5p, thus inhibiting TRAF5/NF-KB pathway.

Though the cause of PD remains to be explored, PD patients were found to manifest reduced TH content, activated microglia, and decreased lactate dehydrogenase (LDH) [21-23]. Interestingly, studies on PD pathogenesis have exhibited that inflammation and oxidative stress causes dopaminergic cell death, which instead facilitated PD inflammation [24]. What's more, the activation of inflammation and oxidative stress could be the biomarkers for the early diagnosis of PD [25]. Thus, inhibiting the inflammation and oxidative stress of PD might bring out direct therapeutic effects for clinical treatment. For instance, niacin [26], epalrestat [27] and bruceine D [28] could ameliorate PD progression via modulating inflammation and oxidative stress. In our study, we also detected the levels of proinflammatory cytokines (including IL$1 \beta, T N F-\alpha$ and IL-6) in the serum of PD patients. It was found that all of IL-1 $\beta \square T N F-a$ and IL- 6 were overexpressed in PD patients compared with those in healthy donors, and the result was consistent with previous studies [29-30].

Multiple evidences have indicated that the aberrant-expressed IncRNAs are involved in the development $\mathrm{PD}$, a positive sign in regulating the neural development, oxidative stress, apoptosis, and neuroinflammation of PD [31-33]. For example, IncRNA-UCA1 is highly expressed in MPP+-induced PD rat brain tissues and SH-SY5Y cells. LncRNA-UCA1 leads to nerve damage and apoptosis by upregulating SNCA [34]. Furthermore, other IncRNAs such as MALAT1 [35], and SNHG14 [36] are all overexpressed in PD and promotes its progression. In this study, our data suggested that SNHG7 is upregulated in PD patients and PD models (both in vivo and in vitro). Interestingly, the high level of SNHG7 had a positive relationship with TNF- $a$ and IL-6 in the serum of PD patients. Functionally, knockdown of SNHG7 expression could not only alleviate rotenone mediated neuronal damage and microglial activation, but also significantly mitigate the levels of inflammatory cytokines and oxidative stress. Therefore, SNHG7 is considered to be a biomarker of PD and also play a role in PD progression via modulate neuronal damage and microglial activation.

MiR-425-5p is a member of miRNAs. Increasing evidences have shown that miR-425-5p are involved in the progression of cancers. For instance, miR-425-5p is overexpressed in gastric cancer (GC) and predicts poor outcome of GC patients [37]. In addition, several studies have indicated that miR-425-5p has powerful effects against inflammation. For example, miR-425-5p was found to be downregulated in LPSinduced sepsis, and enhancing miR-425-5p level markedly relieves the inflammation and septic liver damage via negatively modulating the RIP1-induced necroptosis [38]. Interestingly, miR-425 also exerts a role in central nervous system diseases. For instance, miR-425-5p was significantly downregulated in mild traumatic brain injury (mTBI) at early time-points and identified as a well candidate for diagnosis of mTBI [39]. Besides, miR-425-5p is also considered to be a marker of antidepressant response and regulate MAPK/Wnt-system genes [40]. Interestingly, recent study showed that low level of miR-425 is 
correlated with MPTP induced dopaminergic neuron loss, while miR-425 overexpression obviously attenuated necroptosis activation and dopaminergic neuron loss, and improved locomotor behaviors [41]. Here, our study indicated that miR-425-5p was lowly expressed in PD patients and had negative correlations with the proinflammatory cytokines in the peripheral blood. Moreover, upregulation of miR425-5p showed mighty effects against rotenone induced neuronal damage and microglial inflammations. Therefore, miR-425-5p serves as a promising mediator and therapeutic target for PD.

The IncRNA-miRNA interaction has attracted accumulated attentions, particularly in tumor development and inflammation [42-43]. Interestingly, by conducting bioinformatics analysis, we found that miR-425$5 p$ contains the binding sites with SNHG7. Our gain- and loss- of assays showed that downregulation SNHG7 enhanced miR-425-5p level, while overexpressing SNHG7 led to miR-425-5p inhibition as well significantly reduced the neuroprotective and anti-inflammatory effects of miR-425-5p. Thereby, we proved that the overexpressed SNHG7 could promote neuron damage and microglia activation at least through sponging miR-425-5p.

The TRAF family has been proved to participate in the progress of pathology in PD. For example, triggering receptor expressed on myeloid cells-2 (TREM2), a newly identified receptor expressed on microglia, remarkably reduced MPTP-induced dopaminergic neurodegeneration and neuroinflammation in PD by downregulating the TRAF6/TLR4-mediated activation of the MAPK and NF-KB signaling pathways [44]. In another study, telmisartan has neuroprotective effects in the rotenone rat model of Parkinson's disease through Inhibition of endoplasmic reticulum stress-activated IRE1a-TRAF2-caspase12 apoptotic pathway [45]. TRAF5 is also a vital member of TRAF family. Interestingly, it has been found that TRAF 5 is overexpressed in NeuN- and GFAP-labeled cells in a rat acute spinal cord injury (SCl) model [46]. Moreover, TRAF5 is also a mediator of several CNS diseases such as ischemic brain infarction [47], Huntington's disease [48], and HIV-1 gp120-induced apoptosis in human neurons [49]. In our study, we found that TRAF5 was a target of miR-425-5p by bioinformatics analysis. Downregulating SNHG7 and overexpressing miR-425-5p both inhibited TRAF5 expression in the PD model in vivo and in vitro. Moreover, as an upstream molecule of NF-KB, TRAF5 promotes NF-KB activation, a feature widely recognized by scholars. Accumulated evidence has shown that TRAF5 mediates the activation of NF-KB signaling pathway, and engages in cell apoptosis, the occurrence of inflammation and immune regulation [50-52]. In this study, we found that miR-425-5p inhibited TRAF5, which in turn constrained the activation of the I-KB/NF-KB pathway, thereby reducing inflammation and oxidative stress, and weakening cell apoptosis.

\section{Conclusion}

In summary, this study indicated that SNHG7 was upregulated in PD patients; SNHG7 functioned as a ceRNA by sponging miR-425-5p and promoted TRAF5 mediated inflammation and oxidative stress. SNHG7 low expression or miR-425-5p overexpression profoundly attenuated neuronal apoptosis, inflammation and oxidative stress in PD. Taken together, this research established a novel regulatory axis 
of SNHG7/miR-425-5p/TRAF5/ NF-KB axis in PD. However, in the future, more studies are needed to verify this network in the progression of PD.

\section{Declarations}

\section{Ethics approval and consent to participate}

Our study was approved by the Ethics Review Board of China-Japan Union Hospital of Jilin University.

\section{Consent for publication}

Not applicable.

\section{Availability of data and materials}

The data sets used and analyzed during the current study are available from the corresponding author on reasonable request.

\section{Competing interests}

The authors declare that they have no competing interests.

\section{Funding}

This research did not receive any specific grant from funding agencies in the public, commercial, or notfor- profit sectors.

\section{Acknowledgements}

Not applicable.

\section{Authors contribution}

Jinghui Yang, Methodology and Conceptualization. Shan Yu, Writing - original draft and Supervision . Xueshibojie Liu, Data Curation. Duo Yu, Investigation. Changyong E, Formal analysis and Visulization. All authors read and approved the final manuscript.

\section{References}

1. Gratwicke James J, Marjan F, Thomas. Parkinson's disease dementia: a neural networks perspective. Brain. 2015;138:1454-76.

2. Iciar A-O, Patricia L, Andrew L, et al. Parkinson's disease, insulin resistance and novel agents of neuroprotection. Brain. 2013;136:374-84. 
3. Jiang Tianfang S, Qian C, Shengdi. Oxidative stress: A major pathogenesis and potential therapeutic target of antioxidative agents in Parkinson's disease and Alzheimer's disease. Prog Neurobiol. 2016;147:1-19.

4. Venkatanaidu TSeanHKarri, Nicole T, Rong W, et al. Emerging pathways to neurodegeneration: Dissecting the critical molecular mechanisms in Alzheimer's disease, Parkinson's disease. Biomed Pharmacother. 2019;111:765-77.

5. Wei-Li K, Emma P. Fletcher Michael et al. A novel neuroprotective therapy for Parkinson's disease using a viral noncoding RNA that protects mitochondrial complex I activity.J. Exp. Med. 2012;209:110.

6. Heward James A, Lindsay Mark A. Long non-coding RNAs in the regulation of the immune response. Trends Immunol. 2014;35:408-19.

7. José Luiz,Samblas Mirian,Milagro Marques-Rocha José Luiz,Samblas Mirian,Milagro Fermin I, et al. Noncoding RNAs, cytokines, and inflammation-related diseases. FASEB J, 2015, 29: 3595-611.

8. Zhou Zhiming Z, Yuyin G, Guosheng, et al. Long noncoding RNA SNHG16 targets miR-146a-5p/CCL5 to regulate LPS-induced WI-38 cell apoptosis and inflammation in acute pneumonia. Life Sci. 2019;228:189-97.

9. Chen H, Lan Zedong,Li Qiaomei. et al. Abnormal expression of long noncoding RNA FGD5-AS1 affects the development of periodontitis through regulating miR-142-3p/SOCS6/NF-kB pathway.[J]. Artif Cells Nanomed Biotechnol. 2019;47:2098-106.

10. Ding Xiu-Ming,Zhao Lian-Jiang,Qiao Han-Yong et al. Long non-coding RNA-p21 regulates MPPinduced neuronal injury by targeting miR-625 and derepressing TRPM2 in SH-SY5Y cells. Chem. Biol. Interact., 2019, 307: 73-81.

11. Zhao Jun,Geng Lijiao,Chen Yong. et al. SNHG1 promotes MPP-induced cytotoxicity by regulating PTEN/AKT/mTOR signaling pathway in SH-SY5Y cells via sponging miR-153-3p. Biol Res. 2020;53:1.

12. Qian Chen,Ye Yongyi. Mao Hengxu et al. Downregulated IncRNA-SNHG1 enhances autophagy and prevents cell death through the miR-221/222 /p27/mTOR pathway in Parkinson's disease. Exp Cell Res. 2019;384:111614.

13. Shan Y, Ma J, Pan Y, Hu J, Liu B, Jia L. LncRNA SNHG7 sponges miR-216b to promote proliferation and liver metastasis of colorectal cancer through upregulating GALNT1. Cell Death Dis. 2018;9(7):722.

14. Cheng D, Fan J, Ma Y, et al. LncRNA SNHG7 promotes pancreatic cancer proliferation through ID4 by sponging miR-342-3p. Cell Biosci. 2019;9:28.

15. Zucchelli S, Vilotti S, Calligaris R, et al. Aggresome-forming TTRAP mediates pro-apoptotic properties of Parkinson's disease-associated DJ-1 missense mutations. Cell Death Differ. 2009;16:428-38.

16. Ke N, Pi LH, Liu Q, Chen L. Long noncoding RNA SNHG7 inhibits high glucose-induced human retinal endothelial cells angiogenesis by regulating miR-543/SIRT1 axis. Biochem Biophys Res Commun. 
2019;514(2):503-9.

17. Tian F, Wang J, Zhang Z, Yang J. LncRNA SNHG7/miR-34a-5p/SYVN1 axis plays a vital role in proliferation, apoptosis and autophagy in osteoarthritis. Biol Res. 2020;53(1):9.

18. Grozdanov Veselin,Bliederhaeuser Corinna,Ruf. Wolfgang P, et al. Inflammatory dysregulation of blood monocytes in Parkinson's disease patients. Acta Neuropathol, 2014, 128: 651-63.

19. Song Z, Jin R, Yu S, et al. CD40 is essential in the upregulation of TRAF proteins and NF-kappaBdependent proinflammatory gene expression after arterial injury. PLoS One. 2011;6(8):e23239.

20. Li Fangjun,Li

Xie Y,Li Fangjun,Li Zheng et al. miR-135a suppresses migration of gastric cancer cells by targeting TRAF5-mediated NF-kB activation. Onco Targets Ther, 2019, 12: 975-84.

21. Perlmutter Joel S, Norris Scott A. Neuroimaging biomarkers for Parkinson disease: facts and fantasy. [. J]Ann Neurol. 2014;76:769-83.

22. Balestrino Roberta,Schapira Anthony HV. Glucocerebrosidase and Parkinson Disease: Molecular, Clinical, and Therapeutic Implications. Neuroscientist. 2018;24:540-59.

23. Kordower JH, Olanow C, Warren D, Hemraj B, et al. Disease duration and the integrity of the nigrostriatal system in Parkinson's disease. Brain. 2013;136:2419-31.

24. Xia Yuxing,Funk

Sorrentino Zachary A,Xia Yuxing,Funk Cory, et al. Motor neuron loss and neuroinflammation in a model of rostriatal system in eurodegeneration. Neurobiol Dis, 2018, 120: 98-106.

25. Lotankar S, Prabhavalkar KS, Bhatt LK. Biomarkers for Parkinson's Disease: Recent Advancement. Neurosci Bull. 2017;33(5):585-97.

26. Giri B, Belanger K, Seamon M, et al. Niacin Ameliorates Neuro-Inflammation in Parkinson's Disease via GPR109A. Int J Mol Sci. 2019;20(18):4559.

27. Rahman MM, Chakraborti RR, Potol MA, et al. Epalrestat improves motor symptoms by reducing oxidative stress and inflammation in the reserpine induced mouse model of Parkinson's disease. Animal Model Exp Med. 2019;3(1):9-21.

28. Yang Y, Kong F, Ding Q, Cai Y, Hao Y, Tang B. Bruceine D elevates Nrf2 activation to restrain Parkinson's disease in mice through suppressing oxidative stress and inflammatory response [published online ahead of print, 2020 Apr 19]. Biochem Biophys Res Commun. 2020;S0006-291 × (20)30588-X.

29. Kim R, Kim HJ, Kim A, et al. Peripheral blood inflammatory markers in early Parkinson's disease. J Clin Neurosci. 2018;58:30-3.

30. Qin XY, Zhang SP, Cao C, Loh YP, Cheng Y. Aberrations in Peripheral Inflammatory Cytokine Levels in Parkinson Disease: A Systematic Review and Meta-analysis. JAMA Neurol. 2016;73(11):1316-24.

31. Schmitz Sandra U, Grote Phillip,Herrmann Bernhard G. Mechanisms of long noncoding RNA function in development and disease. Cell Mol Life Sci. 2016;73:2491-509. 
32. Wu Ping,Zuo Xialin,Deng Houliang. et al. Roles of long noncoding RNAs in brain development, functional diversification and neurodegenerative diseases. Brain Res Bull. 2013;97:69-80.

33. Wan Peixing,Su Wenru,Zhuo Yehong,The Role of Long Noncoding. RNAs in Neurodegenerative Diseases[J]Mol Neurobiol. 2017;54:2012-21.

34. Lu M, Sun W-L,Shen J, et al. LncRNA-UCA1 promotes PD development by upregulating SNCA.[J]. Eur Rev Med Pharmacol Sci. 2018;22:7908-15.

35. Chen Q. Huang Xiaoyan,Li Renjie,IncRNA MALAT1/miR-205-5p axis regulates MPP-induced cell apoptosis in MN9D cells by directly targeting LRRK2.[J]. Am J Transl Res. 2018;10:563-72.

36. He-Cheng ZLi-Min,WangMeng-Han,Yang, et al. Dopaminergic neuron injury in Parkinson's disease is mitigated by interfering IncRNA SNHG14 expression to regulate the miR-133b/. a-synuclein pathway[J]Aging (Albany NY). 2019;11:9264-79.

37. Zhang Z, Wen M, Guo J, et al. Clinical value of miR-425-5p detection and its association with cell proliferation and apoptosis of gastric cancer. Pathol Res Pract. 2017;213(8):929-37.

38. Gu C, Hou C, Zhang S. miR-425-5p improves inflammation and septic liver damage through negatively regulating the RIP1-mediated necroptosis. Inflamm Res. 2020;69(3):299-308.

39. Di Pietro V, Ragusa M, Davies D, et al. MicroRNAs as Novel Biomarkers for the Diagnosis and Prognosis of Mild and Severe Traumatic Brain Injury. J Neurotrauma. 2017;34(11):1948-56.

40. Lopez JP, Fiori LM, Cruceanu C, et al. MicroRNAs 146a/b-5 and 425-3p and 24-3p are markers of antidepressant response and regulate MAPK/Wnt-system genes. Nat Commun. 2017;8:15497.

41. Hu YB, Zhang YF, Wang $H$, et al. miR-425 deficiency promotes necroptosis and dopaminergic neurodegeneration in Parkinson's disease. Cell Death Dis. 2019;10(8):589.

42. Sui Y, Lin G, Zheng Y, Huang W. LncRNA MAFG-AS1 boosts the proliferation of lung adenocarcinoma cells via regulating miR-744-5p/MAFG axis. Eur J Pharmacol. 2019;859:172465.

43. Zhang Y, Wang F, Chen G, He R, Yang L. LncRNA MALAT1 promotes osteoarthritis by modulating miR-150-5p/AKT3 axis. Cell Biosci. 2019;9:54.

44. Ren M, Guo Y, Wei X, et al. TREM2 overexpression attenuates neuroinflammation and protects dopaminergic neurons in experimental models of Parkinson's disease. Exp Neurol. 2018;302:205-13.

45. Tong Q, Wu L, Jiang T, Ou Z, Zhang Y, Zhu D. Inhibition of endoplasmic reticulum stress-activated IRE1 a-TRAF2-caspase-12 apoptotic pathway is involved in the neuroprotective effects of telmisartan in the rotenone rat model of Parkinson's disease. Eur J Pharmacol. 2016;776:106-15.

46. Huan W, Wu X, Zhang S, et al. Spatiotemporal patterns and essential role of TNF receptor-associated factor 5 expression after rat spinal cord Injury. J Mol Histol. 2012;43(5):527-33.

47. Wang L, Lu Y, Guan $H$, et al. Tumor necrosis factor receptor-associated factor 5 is an essential mediator of ischemic brain infarction. J Neurochem. 2013;126(3):400-14.

48. Sjögren M, Duarte Al, McCourt AC, Shcherbina L, Wierup N, Björkqvist M. Ghrelin rescues skeletal muscle catabolic profile in the R6/2 mouse model of Huntington's disease. Sci Rep. 2017;7(1):13896. 
49. Chen W, Tang Z, Fortina P, et al. Ethanol potentiates HIV-1 gp120-induced apoptosis in human neurons via both the death receptor and NMDA receptor pathways. Virology. 2005;334(1):59-73.

50. WuFeng,YaoDong-Sheng,Lan Tian-Ying, et al. Berberine prevents the apoptosis of mouse podocytes induced by TRAF5 overexpression by suppressing NF-KB activation.[J].Int. J. Mol. Med., 2018, 41: 555-563.

51. Tao Tao,Cheng Chun,Ji Yuhong. et al. Numbl inhibits glioma cell migration and invasion by suppressing TRAF5-mediated NF-KB activation.[J].Mol. Biol Cell. 2012;23:2635-44.

52. Xie Y,Li Fangjun,Li Zheng et al. miR-135a suppresses migration of gastric cancer cells by targeting TRAF5-mediated NF-KB activation.[J].Onco Targets Ther, 2019, 12: 975-984.

\section{Figures}

A

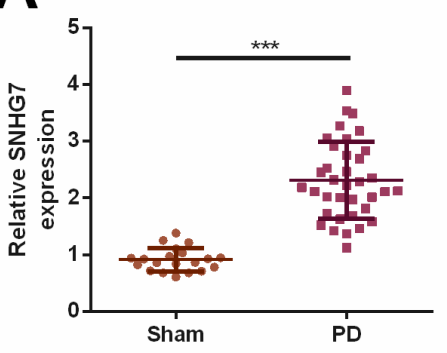

$\mathbf{E}$

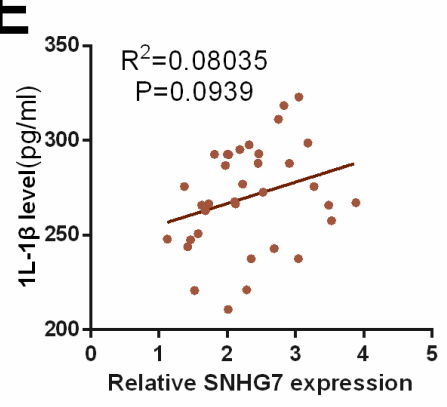

$\mathbf{F}$

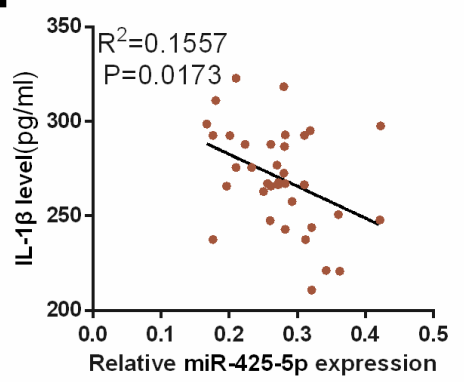

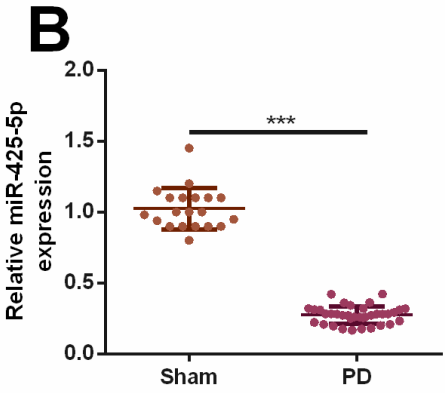
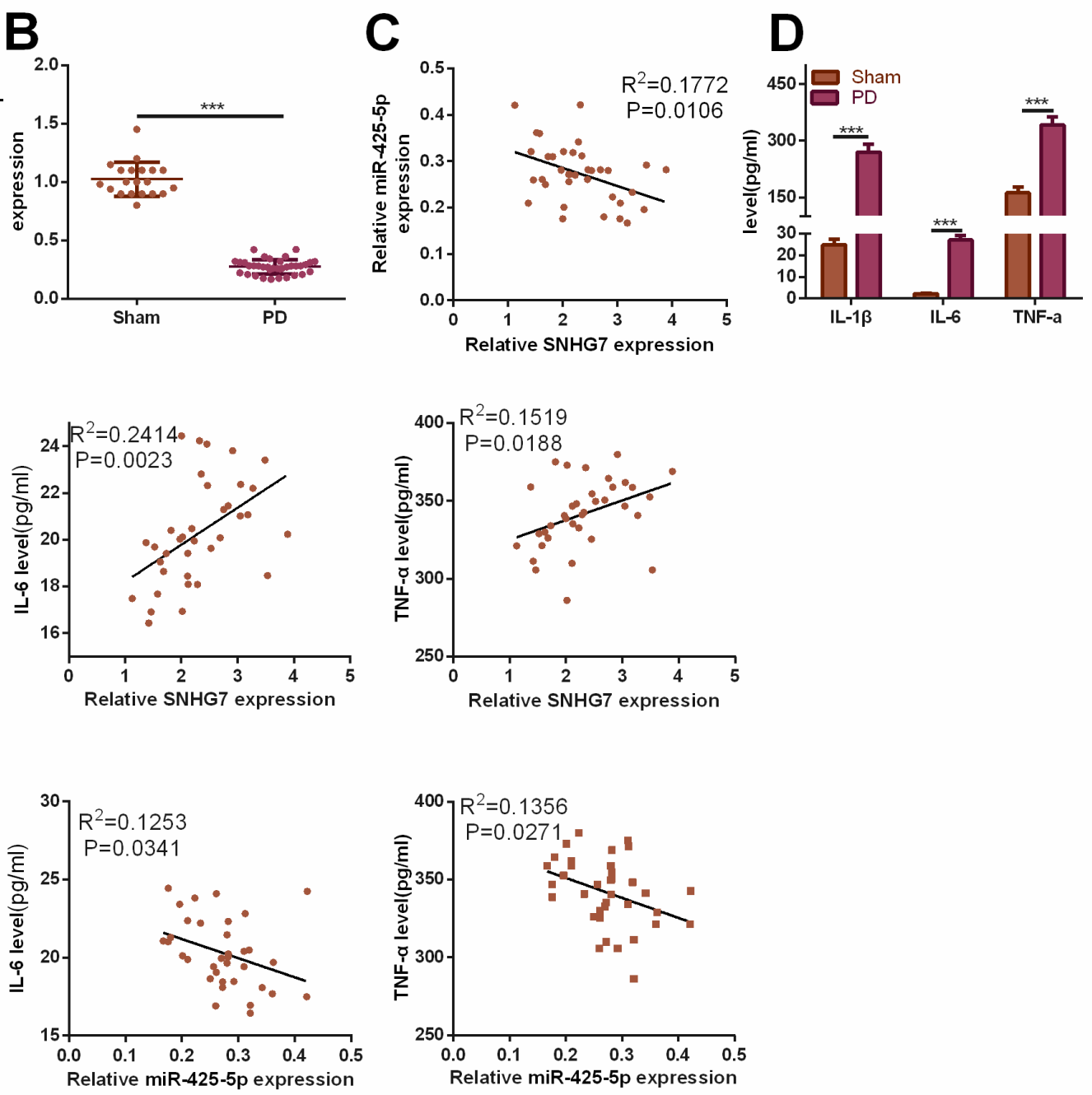

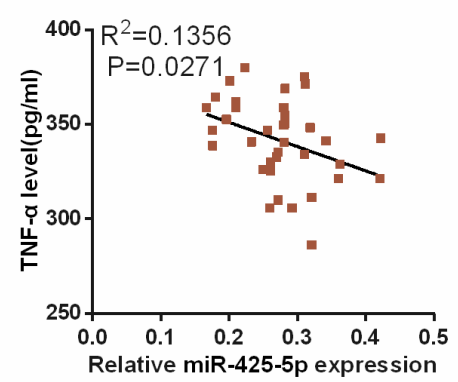

\section{Figure 1}

Expression characteristics of SNHG7 and miR-425-5p in PD patients. (A, B) The relative expression of SNHG7 and miR-425-5p in the plasma of healthy donors and PD patients was detected by qRT-PCR. (C) 
Pearson correlation analyzed the correlation between SNHG7 and miR-425-5p in PD patients' plasma. (D) ELISA was applied to measure the IL-1 $1 \beta$, IL- 6 and TNF-a relative expressions in the plasma of patients and healthy controls. (E) Pearson correlation test analyzed the correlation between SNHG7 and IL-1 $1 \beta$, IL-6, TNF- $\alpha$ in the plasma of PD patients. (F) Pearson correlation test analyzed the correlation between miR425-5p and IL-1 $\beta$, IL-6, TNF- $\alpha$ in the plasma of PD patients. ${ }^{* * *} \mathrm{P}<0.001$.
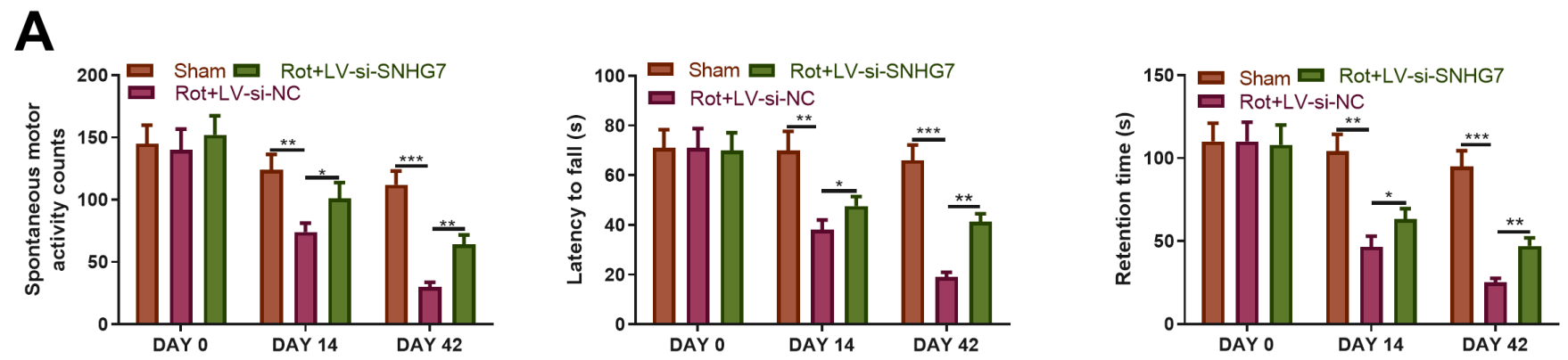

B
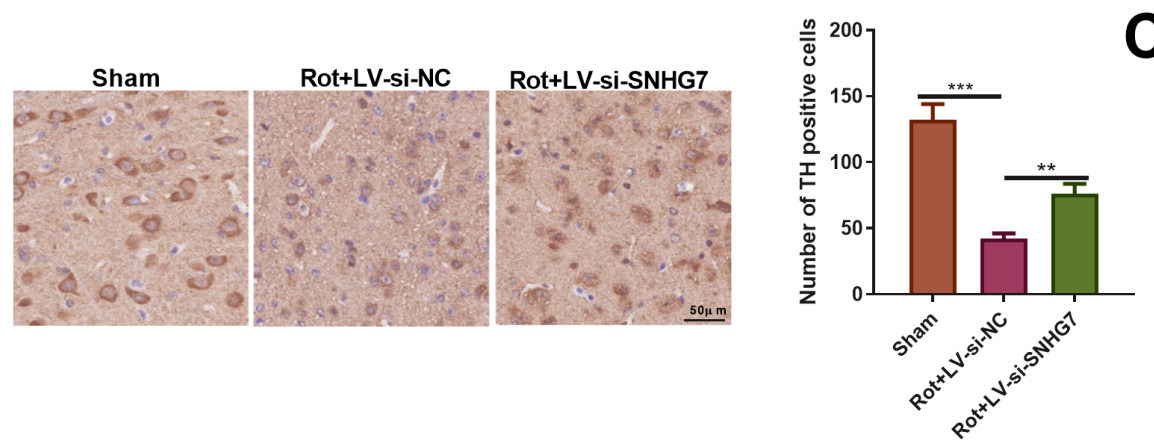

D
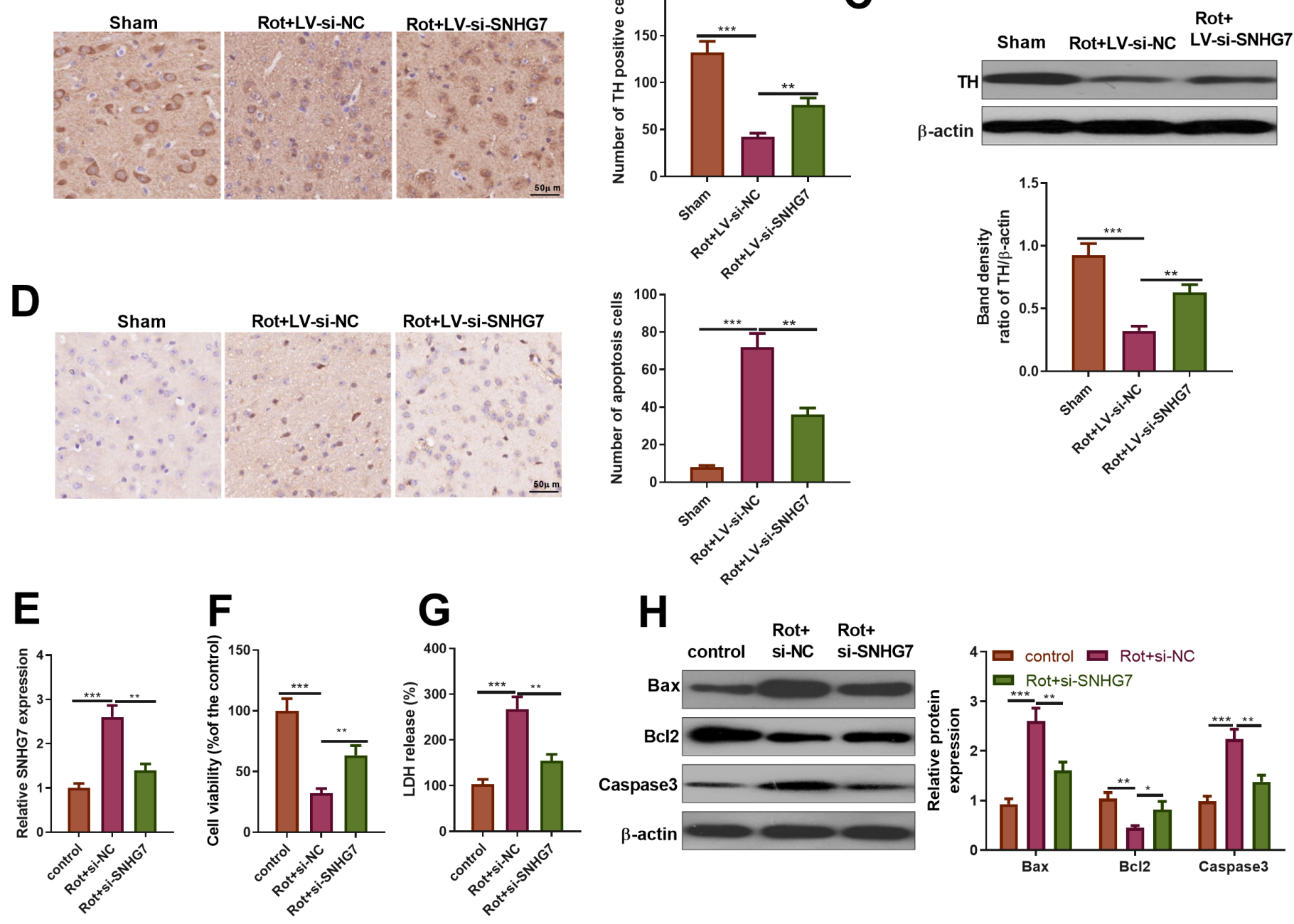

Figure 2

SNHG7 inhibition attenuated neuronal apoptosis in PD models in vivo and in vitro. (A) The spontaneous locomotor activity test, carousel experiment and bevel experiment were used to comprehensively evaluate 
the rat's motor skills. (B) Immunohistochemistry was utilized to detect the number of tyrosine hydroxylase (TH) positive cells in the substantia nigra (SN) area of rats. (C) Western blot was employed to detect the $\mathrm{TH}$ relative expression in rat $\mathrm{SN}$ region. (D) TUNEL staining was used to detect the apoptotic neuron number in rats. (E) SH-SY5Y cells with low SNHG7 expression were constructed, and qRT-PCR was used to detect the SNHG7 relative expression. (F) MMT detected neuronal proliferation. (G) ELISA assay was used to detect LDH levels of cells in each group. $(\mathrm{H})$ The relative expression of apoptotic proteins: Bax, $B c l 2$ and Caspase 3 was detected by western blot assay. \&\& $P<0.01, \& \& \& P<0.001$ (vs Sham); $P<0.05$, ** $\mathrm{P}<0.01, \star \star \star \mathrm{P}<0.001$.
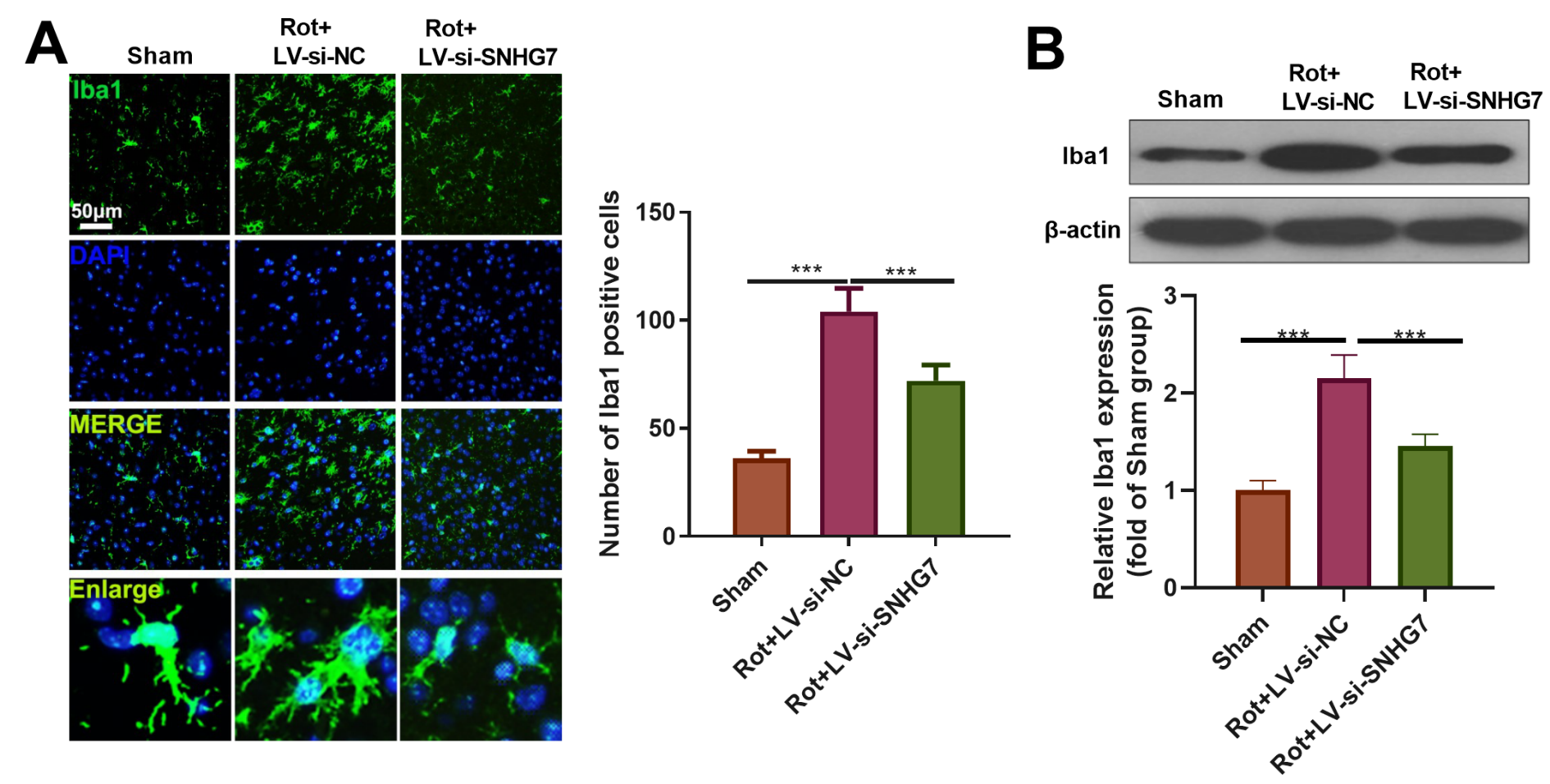

Figure 3

SNHG7 inhibition attenuated Iba1 labeled microglia activation in the rat PD models. (A). Immunofluorescence was used to detect Iba1 labeled microglia in the SN area. (B) Western blot was conducted to detect Iba1 expression in the $\mathrm{SN}$ area. ${ }^{\star \star *} \mathrm{P}<0.001$. 
A

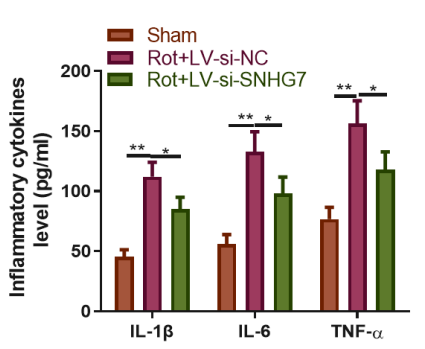

D

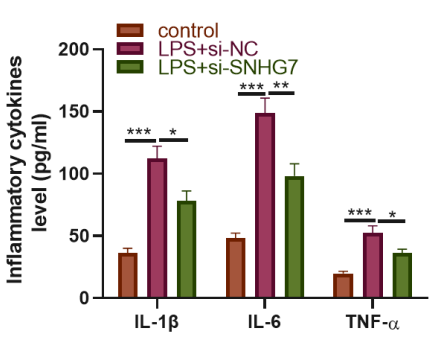

B

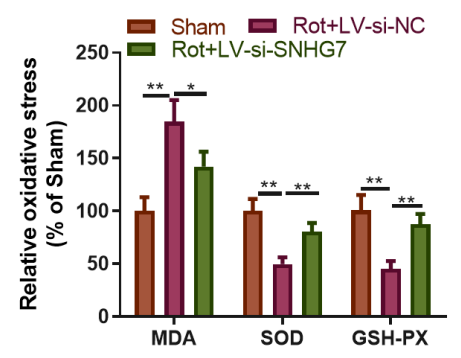

E

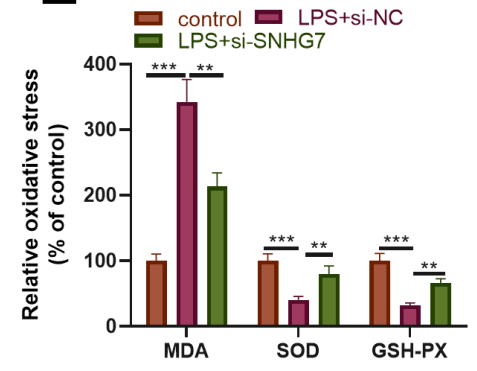

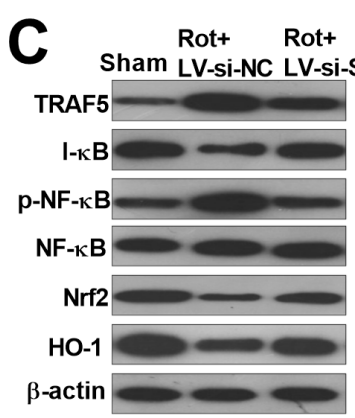

$\mathbf{F}$

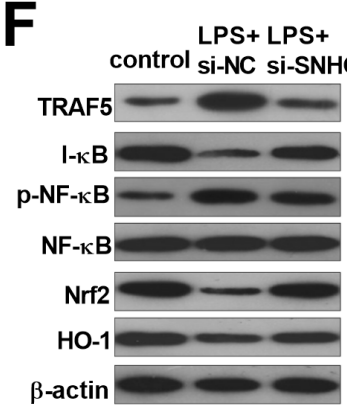

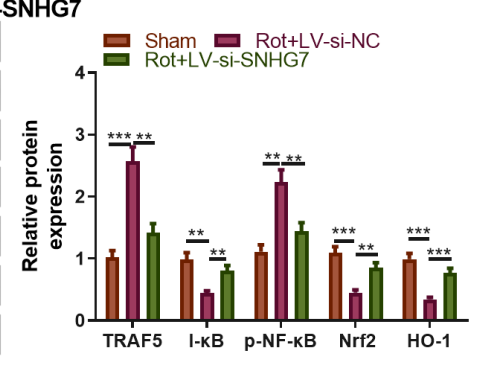

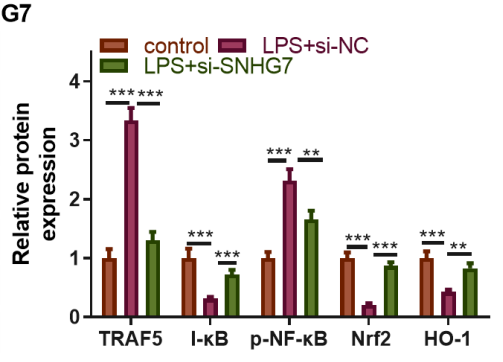

Figure 4

SNHG7 inhibition mitigated inflammation and oxidative stress in PD models. (A) ELISA was utilized to detect the IL-1 $\beta$, IL- 6 and TNF- $\alpha$ expressions in SD rat brain tissue. (B) The oxidative stress related factors (including MDA, SOD, and GSH-PX) in brain tissues were detected. (C) Western blot was used to detect the relative expression of TRAF5, I-KB, NF-kB, Nrf2, HO-1 in the SN area. (D) The expressions of inflammatory factors IL-1 $\beta$, IL-6 and TNF- $\alpha$ in BV2 cells were detected. (E) ELISA was used to detect the expression of inflammatory oxidative stress factors: MDA, SOD and GSH-PX in BV2 cells. (F) Western blot was carried

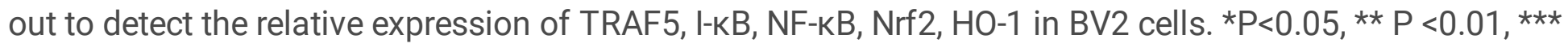
$\mathrm{P}<0.001$. 
A B

WT (SNHG7) 5' cugccgugucacAUCUGUGUCAUu 3'

miR-425-5p 3' aguugcccucacUAG-CACAGUAa 5' MUT (SNHG7) 5' cugccgugucacCAUUUGCAUAUU 3'

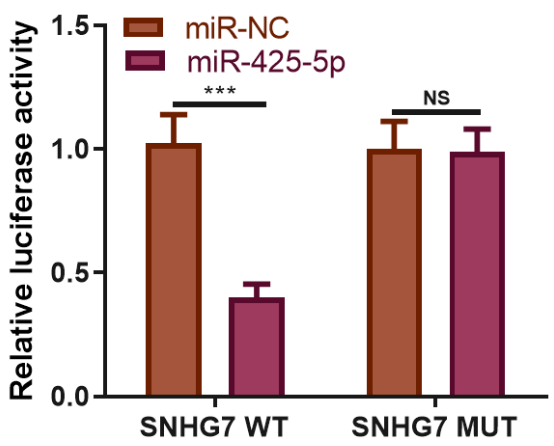

E
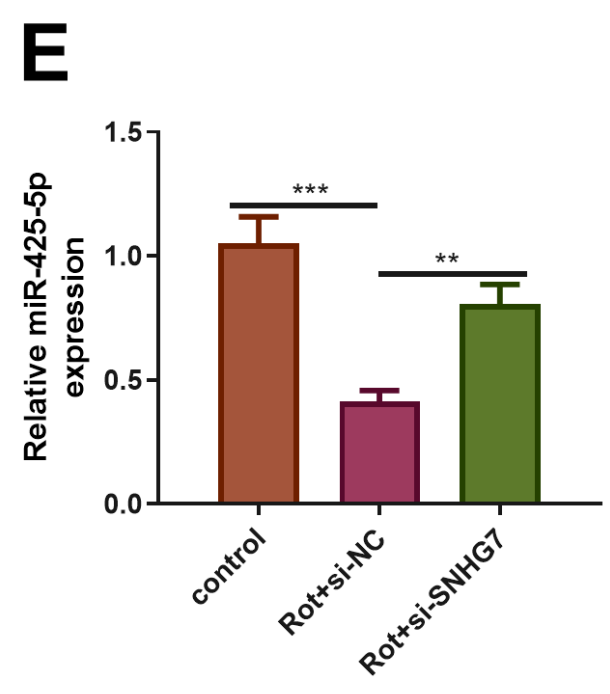

C

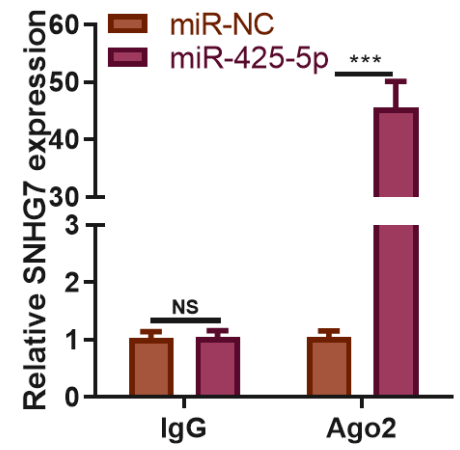

D
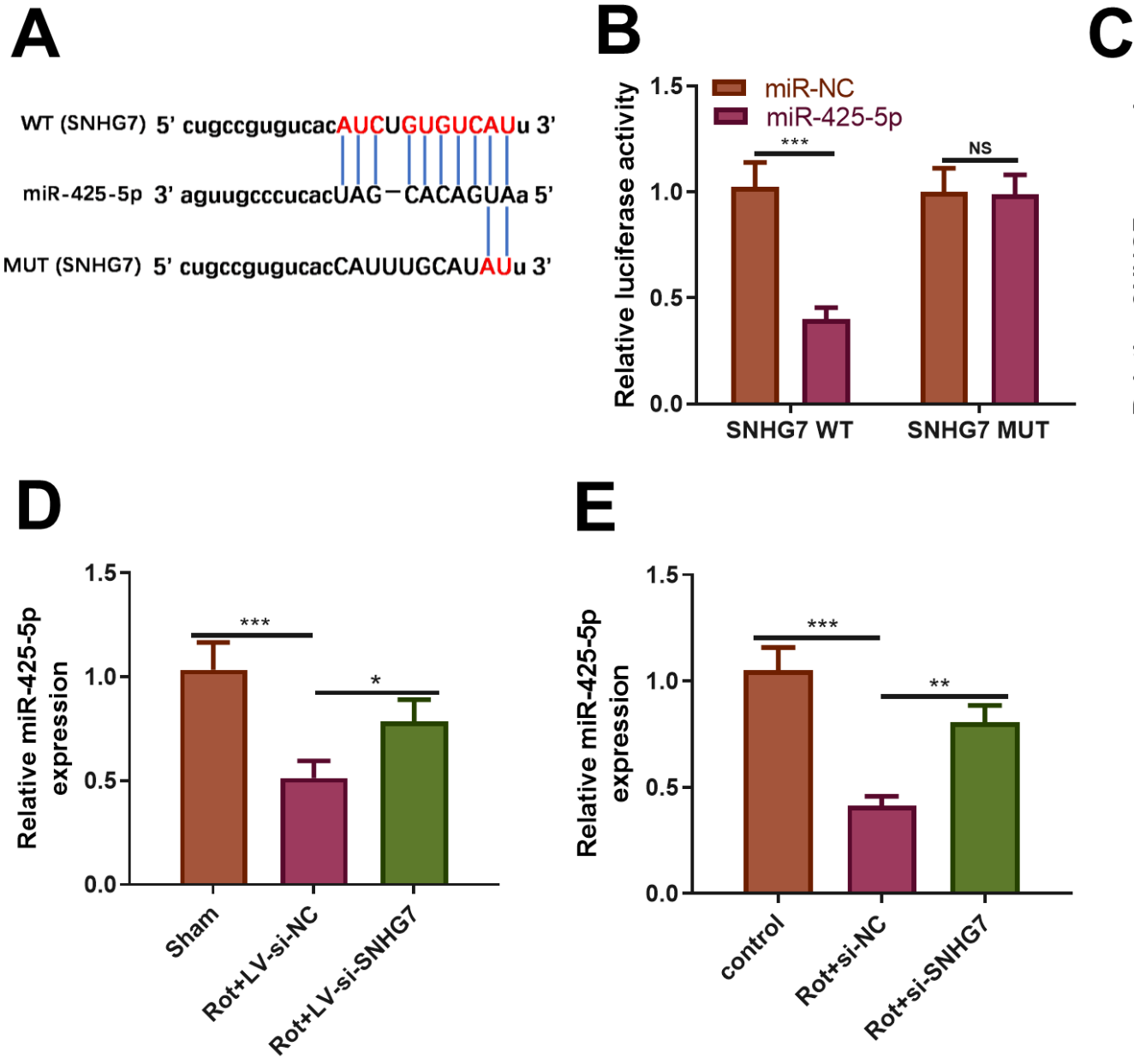

D

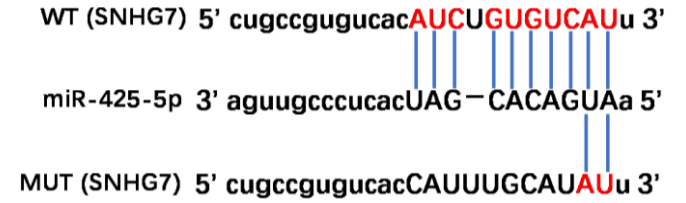

Figure 5

SNHG7 targeted miR-425-5p. (A) The online database Starbase (http://starbase.sysu.edu.cn/) was used to predict the binding sites between SNHG7 and miR-425-5p. (B, C) The luciferase reporter assay (B) and RIP assay (C)were conducted in BV2 cells to further verify the targeting relationship between SNHG7 and miR-425-5p. (D, E) The miR-425-5p relative expression was detected the PD model in vivo (D) and in vitro (E) by $q R T-P C R$, respectively. NS $P>0.05, * P<0.05, * \star P<0.01$, $* \star * P<0.001$. 

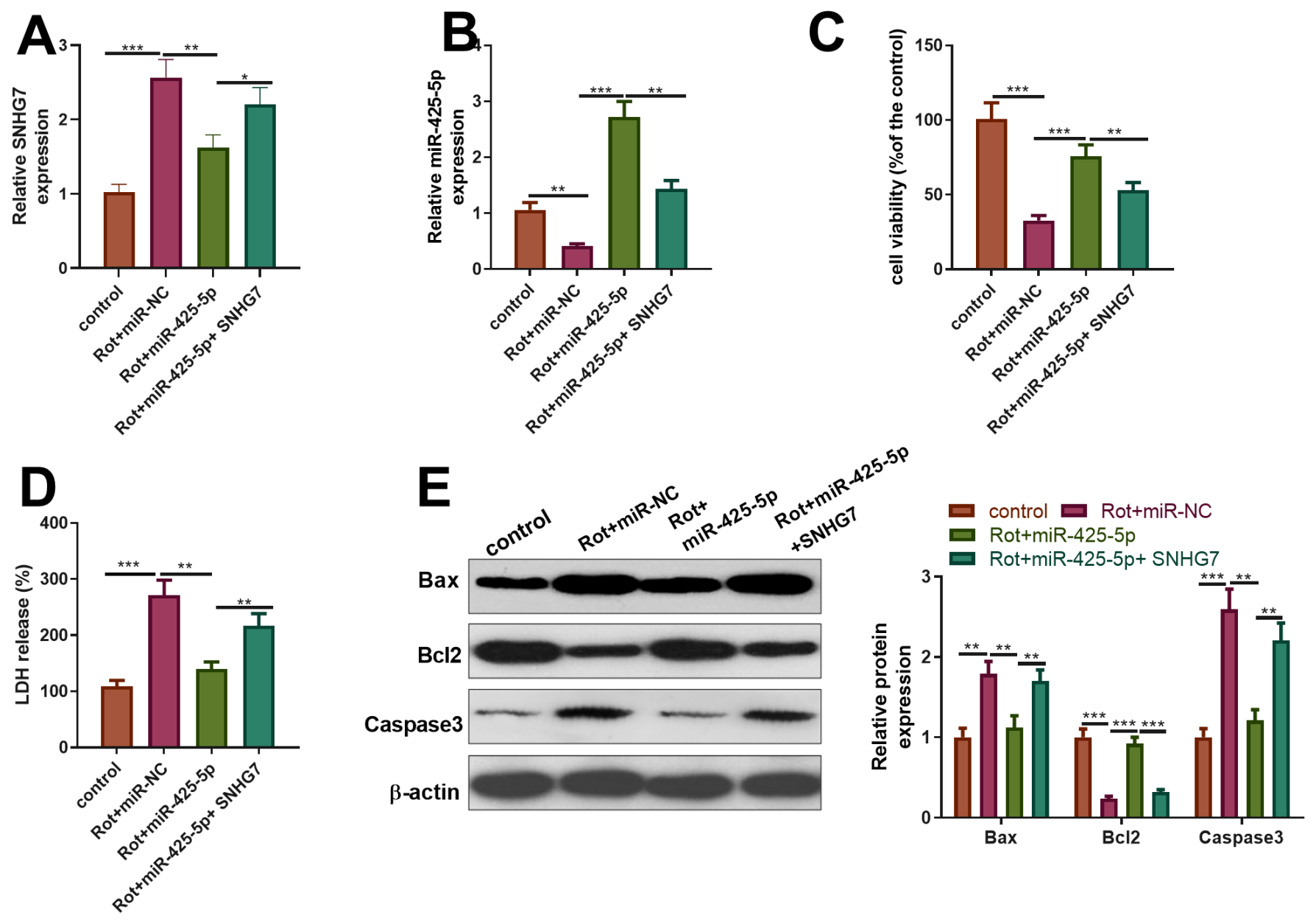

Figure 6

SNHG7 overexpression inhibited the neuroprotective effects of miR-425-5p on neurons. SH-SY5Y cells were transfected miR-425-5p mimics and/or SNHG7 overexpressing plasmids and then subjected to rotenone (500 nM). (A, B) The SNHG7 and miR-425-5p relative expressions in each group of SH-SY5Y cells was detected via qRT-PCR. (C) MTT was for detection of neuron viability. (D) ELISA was used to detect LDH levels. (E) Western blot was used for detecting the apoptosis-related proteins' expressions (Bax, Bcl2, and Caspase3) in each group. ${ }^{*} \mathrm{P}<0.05$, ${ }^{\star *} \mathrm{P}<0.01$, $* \star * \mathrm{P}<0.001$. 
A

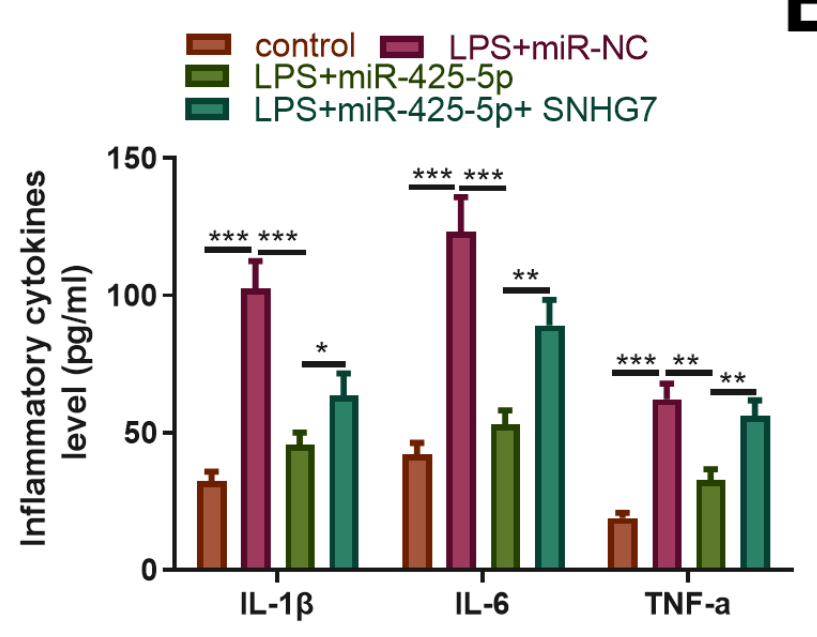

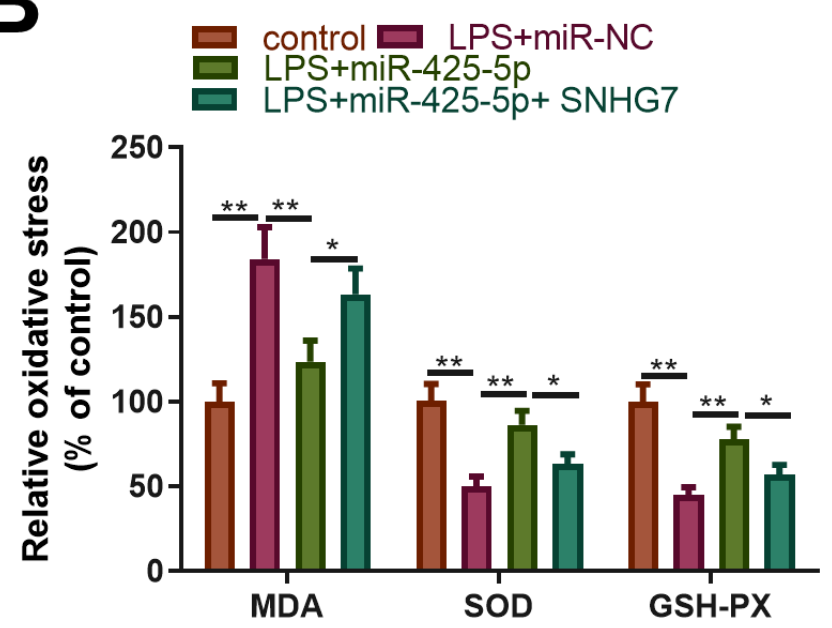

C
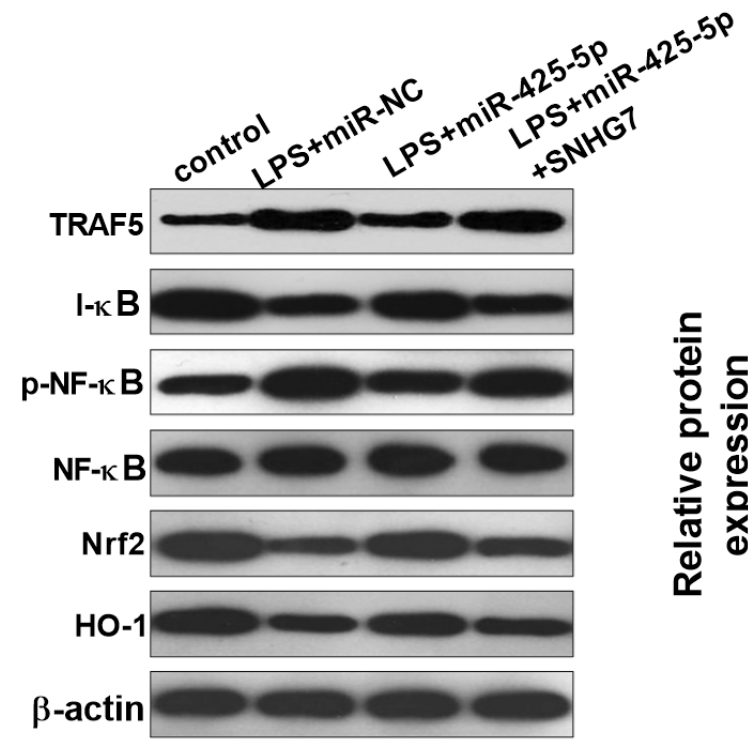

D

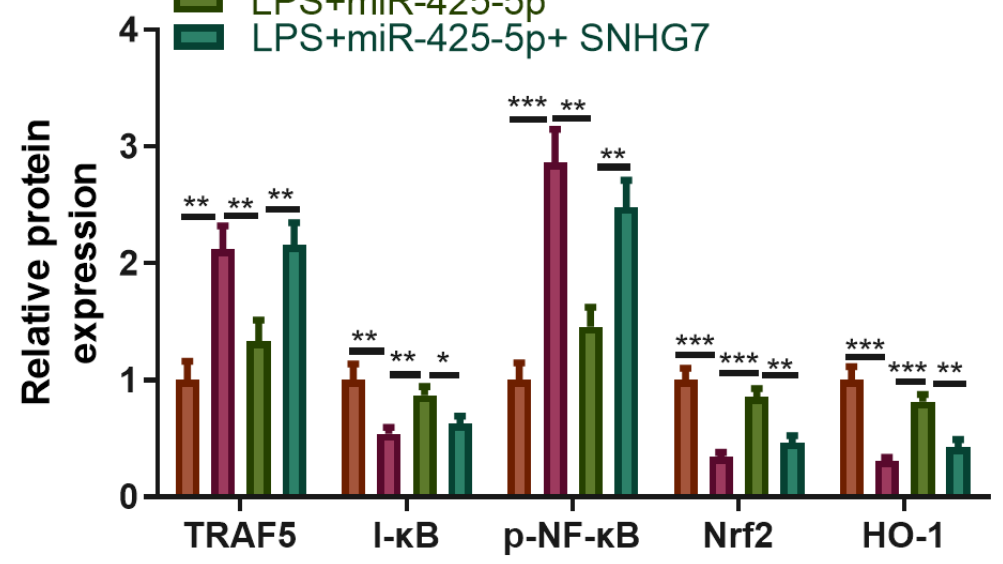

Figure 7

SNHG7 promoted TRAF5/NF-KB activation through competitively binding to miR-425-5p. BV2 cells were transfected miR-425-5p mimics and/or SNHG7 overexpressing plasmids and then subjected to LPS (10 $\mu \mathrm{g} / \mathrm{mL}$ ). (A) ELISA was used to estimate the relative expression of inflammatory factors: IL-1 13 , IL-6 and TNF-a. (B) ELISA was applied detecting the relative expression of oxidative stress factors: MDA, SOD and GSH-PX. (C, D) Western blot was used for detecting the expression of TRAF5, l-kB, NF-kB, Nrf2, HO-1 in each group. ${ }^{*} P<0.05$, ** $P<0.01$, *** $P<0.001$. 
A

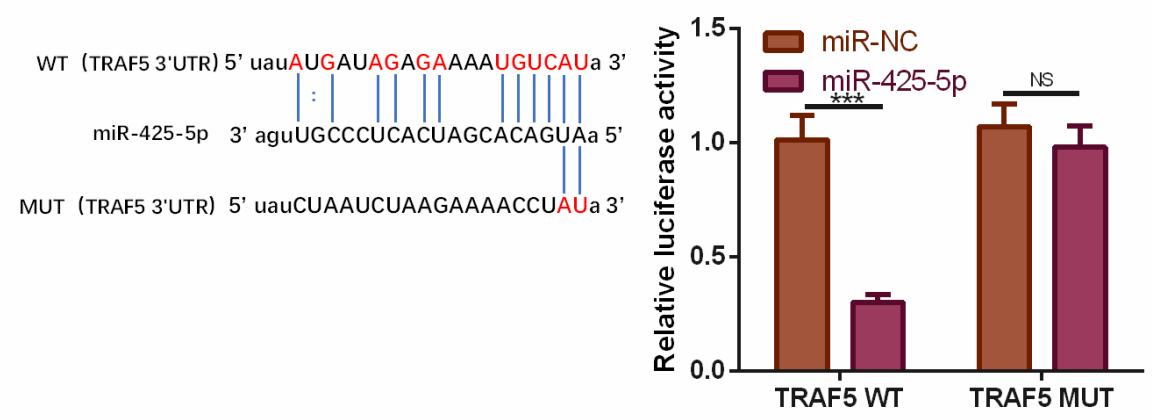

C

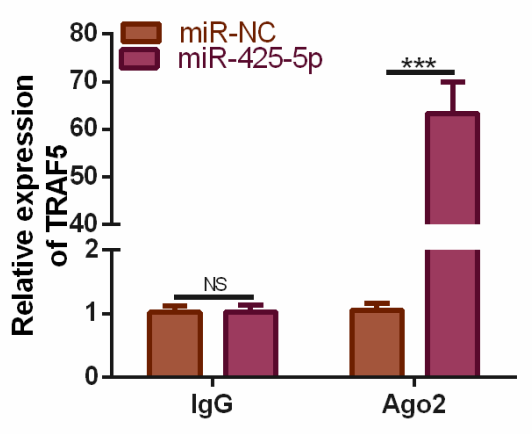

\section{Figure 8}

miR-425-5p targeted TRAF5. (A) The online database Starbase (http://starbase.sysu.edu.cn/) was used to predict the binding site between miR-425-5p and TRAF5. (B, C) In SH-SY5Y cells, luciferase reporter assay and RIP assay were used to further verify the targeting relationship between miR-425-5p and TRAF5. NS $P>0.05, \star \star \star ~ P<0.001$. 